East African Medical Journal Vol. 83 No. 1 January 2006

HAEMATOLOGICAL, LIPID PROFILE AND OTHER BIOCHEMICAL PARAMETERS IN NORMAL AND HYPERTENSIVE SUBJECTS AMONG THE POPULATION OF THE EASTERN PROVINCE OF SAUDI ARABIA

F.A. Al-Muhana, MD, E.B. Larbi, PhD, FRCP, Department of Internal Medicine, A.K. Al-Ali, BSc, PhD, Department of Clinical Biochemistry, A. Al-Sultan, MD, FRCPC, Department of Internal Medicine, S. Al-Ateeeq, BSc, MSc, Department of Clinical Biochemistry, L. Soweilem, MD, FFCM, Department of Family and Community Medicine, L. Goa, MD, FRCSC, Department of Internal Medicine, A.A. Bahnassy, MSc, PhD, Department of Family and Community Medicine, A. Al- Rubaish, MD and M.F. Abdulmohsen, MD, Department of Internal Medicine, College of Medicine, King Faisal University, P.O. Box 2114, Dammam 31451, Saudi Arabia

Request for reprints to: Prof. A.K. Al-Ali, Dean, College of Medicine, King Faisal University, P.O. Box 2114, Dammam, 31451, Saudi Arabia

\title{
HAEMATOLOGICAL, LIPID PROFILE AND OTHER BIOCHEMICAL PARAMETERS IN NORMAL AND HYPERTENSIVE SUBJECTS AMONG THE POPULATION OF THE EASTERN PROVINCE OF SAUDI ARABIA
}

\author{
F.A. AL-MUHANA, E.B. LARBI, A.K. AL-ALI, A. AL-SUltAn, S. AL-ATEEEQ, L. SOWEILEM, \\ L. GOA, A.A. BAHNASSY, A. AL-RUBAISH and M.F. ABDULMOHSEN
}

\begin{abstract}
Objective: To determine the lipid profile complete blood count and other biochemical parameters in normotensive and hypertensive individuals.

Design: Cross-sectional population-based epidemiological household survey.

Setting: Population sample of the Eastern Province of Saudi Arabia.

Results: Hypertensive individuals had significantly higher mean levels of glucose, Tc, LDL-c, HDL-c, triglycerides and HBAIc, compared to normotensive individuals while there were no significant difference in the mean levels of Apo AI and Apo B. Within the same group there were variations in the levels of certain parameters between male and female. While the mean levels of haemoglobin, WBC and platelets were significantly higher in the hypertensive group compared to normotensive, there were no significant differences between these two groups in the levels of RBC, MCV, HCT, MCH and MCHC. However, the mean levels haemoglobin, RBC count and HCT were significantly higher in male compared to female within the same group with no significant difference in levels of WBC, MCV, MCH and MCHC. Furthermore, the mean concentration of platelets was significantly higher in females compared to male within the same group. Hypertensive individuals had significantly higher serum sodium, chloride and calcium levels but a significantly lower potassium level when compared to normotensive with no siginificant differences between male and female within the same group.

Conclusion: The lipid and electrolyte profile of hypertensive individuals differ from that of normotensive individuals in this population. This study has contributed towards establishing the normal values for a number of parameters involved in the aetiology of cardiovascular diseases in the population of Eastern province.
\end{abstract}

\section{INTRODUCTION}

Cardiovascular disease (CVD) is the leading cause of illness in the industrial world and its incidence is progressively increasing in the developing countries(1). Hypertension is the most important risk factor of CVD which increases the risk of stroke, myocardial infarction, heart and renal failure. Coronary atherosclerosis is the result of complex processes which include inflammatory processes, low density lipoprotein (LDL) accumulation and smooth muscle cell migration (2). These processes at different degrees are modulated by many factors including hypertension, diabetes and smoking (3). It has well been established that high blood LDL cholesterol (LDL-c) among others, is associated with the early development of atheroselerotic plaques. An increase of one standard deviation for every LDL cholesterol concentration has been shown to be associated with
5.4\% more atherosclerotic lesions (4). Additionally, biochemical research has provided evidence that LDL cholesterol promotes atherosclerotic calcification of vascular cells due to products of lipid oxidation (5).

A number of studies have reported a progressive increase in the incidence of CVDs in the Mediterranean region including Saudi Arabia (6,7). This has been attributed to the tremendous changes in socio-economic factors and the life style of these populations. This changing life style and technology of the modern environment which brings with them diminished physical activity, abundance of high energy and dense food in combination with social stresses and deleterious habits in populations unaccustoned to such exposure are leading to increasing occurence of CVDs. Yet, clinical trials have provided compelling evidence that coronary heart disease is largely preventable (8). However the efficiency and success of the above 
measures depend on previous identification of the population risk factors.

There have been many studies in the kingdom of Saudi Arabia concerning hypertension and other risk factors, with varied results and conclusion (9-14). This may reflect the non uniformity of the study design populations surveyed and different laboratory procedures. In addition many of the studies are hospital based (15).

The present study was carried out to establish the local norms and identify the risk factors in the communities of the Eastern Province as part of a comprehensive population based study of hypertension.

\section{MATERIALS AND METHODS}

During a household screening programme of hypertension in the Eastern province of Saudi Arabia, 250 hypertensive Saudi males and females aged 18-60 and similar numbers of controls were randomly selected. The section was from a sample of 6000 representing the population of Eastern Province with all its socio-economical and genetic variabilities. A written informed consent was obtained from each subject. Extreme care was taken in the random selection of hypertensive and control groups to ensure that they represented a broad cross sectional coverage of all population in the Eastern Province. The control group consisted of healthy volunteers who were not taking hormones or drugs known to affect plasma lipid levels. Hypertension was considered to be present if subjects met the following criteria: Systolic pressure of $140 \mathrm{mmHg}$ and above and diastolic pressure of $90 \mathrm{mmHg}$ and above on two occasions by the same interviewer (1). Blood samples were obtained at the local primary health centre after an overnight fast. Blood pressure measurements were obtained using a mercury sphygmomanometer on three occations during the interview. Blood collected in red top vacutainer tubes was allowed to stand for 30 minutes at room temperature to allow complete clotting and clot retraction. Samples were centrifuged at 2000 $\mathrm{x} \mathrm{g}$ for $15 \mathrm{~min}$, divided into aliquots and stored at $-20^{\circ}$ celsius. Three millilitres of blood was collected in EDTA coated tubes and $4 \mathrm{ml}$ of blood was collected in plain tubes. Haematological indices were determined for all samples using a haematological analyzer (ACT-8 coulter Electronies). Glucose, creatinine, BUN, uric acid, Tc, HDL-c and triglycerides were determined as previously described (17-19). LDL-c was calculated using the Friedwald formula (20). Apo A-I and Apo B were determined by ELIZA methods while HBAI-c was determined by affinity chromatography (Bio Merieux Sa, Immunoturbidimetric assay of Apo A-I and Apo B, Helena GLYCO-TEK Affinity Column). Serum sodium, potassium, chloride and calcium were determined using electrolyte analyzer (AVL 9180 Electrolyte analyzer). Mean values in lipid lipoproteins and other parameters between subgroups were compared using the independent sample t-test. All statistical analyses were perfomed using the SPSS package (version 10). A p-value of $<0.05$ was considered to be statistically significant. Quality control procedures included the re-analysis of $5 \%$ of frozen specimens randomly selected either within assay or between assays.

\section{RESULTS}

The mean values of blood indices are presented in Table 1. Within the hypertensive and normotensive groups, the mean levels of haemoglobin, HCT and RBC were significantly higher in male compared to females $(p<0.05)$. However, the platelet level was higher in female than in male $(\mathrm{p}<0.05)$. There were no significant differences in the levels of $\mathrm{WBC}, \mathrm{MCV}, \mathrm{MCH}$ and $\mathrm{MCHC}$ between males and female $(\mathrm{p}>0.05)$. While the mean levels of haemoglobin, $\mathrm{WBC}$ and platelets were significantly higher in the hypertensive group compared to normotensive $(\mathrm{p}<0.05)$, there were no significant differences between these two groups in the levels of $\mathrm{RBC}, \mathrm{MCV}, \mathrm{HCT}, \mathrm{MCH}$ and $\mathrm{MCHC}(\mathrm{p}>0.05)$. Hypertensive individuals had significantly higher mean levels of glucose, Tc, LDL-c, HDL-c, triglycerides and HbAIc compared to normotensive individuals, while there were no significant differences in the mean levels of Apo AI and Apo B. However, there were variations in the levels of certain parameters between male and female within the hypertensive and the normotensive groups. The most striking difference was in the level of $\mathrm{Tg}$ where the male had a signicantly higher mean $\mathrm{Tg}$ level compared to female in both groups.

Table 1

$C B C($ Mean $\pm S D)$ of normotensive controls and hypertensive groups

\begin{tabular}{|c|c|c|c|c|c|c|c|c|}
\hline Gender & $\begin{array}{l}\text { WBC } \\
\left(10^{9} / \mathrm{L}\right)\end{array}$ & $\begin{array}{c}\mathrm{RBC} \\
\left(10^{12} / \mathrm{L}\right)\end{array}$ & $\begin{array}{l}\text { Haemoglobin } \\
(\mathrm{g} / \mathrm{dl})\end{array}$ & $\begin{array}{l}\mathrm{HCT} \\
(\%)\end{array}$ & $\begin{array}{l}\mathrm{MCV} \\
\text { (fl) }\end{array}$ & $\begin{array}{c}\mathrm{MCH} \\
(\mathrm{pg})\end{array}$ & $\begin{array}{l}\mathrm{MCHC} \\
(\mathrm{g} / \mathrm{dl})\end{array}$ & $\begin{array}{c}\text { PLT } \\
\left(10^{9} / \mathrm{L}\right)\end{array}$ \\
\hline \multicolumn{9}{|l|}{ Controls } \\
\hline Male & $5.64 \pm 1.53$ & $5.08 \pm 0.59$ & $13.74 \pm 1.44$ & $39.99 \pm 3.57$ & $79.20 \pm 7.36$ & $27.16 \pm 3.30$ & $34.18 \pm 1.23$ & $237.8 \pm 57.7$ \\
\hline Female & $5.64 \pm 1.55$ & $4.49 \pm 0.55$ & $11.77 \pm 1.30$ & $34.78 \pm 3.18$ & $77.66 \pm 8.39$ & $26.63 \pm 3.82$ & $33.99 \pm 1.31$ & $275.90 \pm 69.1$ \\
\hline Total & $5.64 \pm 1.54$ & $4.82 \pm 0.65$ & $12.84 \pm 1.69$ & $37.70 \pm 4.27$ & $78.52 \pm 7.85$ & $26.93 \pm 3.54$ & $34.10 \pm 1.27$ & $254.6 \pm 65.7$ \\
\hline \multicolumn{9}{|c|}{ Hypertensives } \\
\hline Male & $6.41 \pm 1.17$ & $5.04 \pm 0.48$ & $14.26 \pm 1.38$ & $41.91 \pm 3.50$ & $83.55 \pm 6.53$ & $28.43 \pm 2.70$ & $34.10 \pm 1.04$ & $264.53 \pm 64.8$ \\
\hline Female & $6.42 \pm 1.44$ & $4.59 \pm 0.49$ & $12.18 \pm 1.27$ & $36.43 \pm 3.66$ & $79.40 \pm 7.74$ & $26.58 \pm 3.16$ & $33.45 \pm 0.99$ & $288.0 \pm 75.4$ \\
\hline Total & $6.42 \pm 1.45$ & $4.79 \pm 0.53$ & $13.11 \pm 1.67$ & $38.83 \pm 4.50$ & $81.23 \pm 7.51$ & $27.40 \pm 3.04$ & $33.73 \pm 1.05$ & $277.7 \pm 71.7$ \\
\hline
\end{tabular}


Table 2

Serum lipid profile, glucose and HbAIc of the two study groups (Mean $\pm S D$ )

\begin{tabular}{lcccccr}
\hline \multirow{2}{*}{ Test Parameters } & \multicolumn{3}{c}{ Controls } & & & Hypertensives \\
\cline { 2 - 7 } & Male & Female & Total & Male & Female & Total \\
\cline { 2 - 7 } & & & & & \\
Glucose (mg/dL) & $93.84 \pm 47.8$ & $89.1 \pm 42.7$ & $91.15 \pm 45.3$ & $136.5 \pm 60.5$ & $122.5 \pm 52.0$ & $128.8 \pm 56.3$ \\
TC (mg/dL) & $168.8 \pm 30.64$ & $176.4 \pm 38.63$ & $172.7 \pm 34.88$ & $196.7 \pm 33.46$ & $195.7 \pm 40.18$ & $196.1 \pm 37.24$ \\
LDL-c (mg/dL) & $105.8 \pm 25.6$ & $114.1 \pm 30.5$ & $109.8 \pm 28.3$ & $126.7 \pm 33.4$ & $131.4 \pm 36.7$ & $129.2 \pm 35.2$ \\
Tg (mg/dL) & $104.8 \pm 67.2$ & $89.4 \pm 55.3$ & $97.4 \pm 62.2$ & $145.2 \pm 76.9$ & $119.4 \pm 68.2$ & $131.0 \pm 73.2$ \\
HDL-g (mg/dL) & $40.7 \pm 8.5$ & $44.6 \pm 9.1$ & $42.6 \pm 9.0$ & $44.1 \pm 8.7$ & $46.6 \pm 9.0$ & $45.7 \pm 8.9$ \\
Apo Al (g/L) & $1.53 \pm 0.35$ & $1.63 \pm 0.36$ & $1.58 \pm 0.36$ & $1.45 \pm 0.31$ & $1.60 \pm 0.55$ & $1.52 \pm 0.44$ \\
Apo B (g/L) & $1.05 \pm 0.33$ & $1.00 \pm 0.32$ & $1.03 \pm 0.32$ & $1.06 \pm 0.24$ & $0.93 \pm 0.32$ & $1.0 \pm 0.29$ \\
HbAlc (\%) & $7.48 \pm 2.24$ & $7.45 \pm 2.4$ & $7.47 \pm 2.31$ & $8.99 \pm 2.98$ & $8.93 \pm 2.3$ & $8.96 \pm 2.3$
\end{tabular}

Table 3

Serum Electrolyte, BUN, creatinine and uric acid levels of the two study groups (Mean $\pm S D$ )

\begin{tabular}{lcccccr}
\hline & \multicolumn{3}{c}{ Normotensive Controls } & & Hypertensives \\
\cline { 2 - 7 } Test parameters & Male & Female & Total & Male & Female & Total \\
\hline Sodium (meq/L) & $132.8 \pm 13.5$ & $131.5 \pm 12.1$ & $132.1 \pm 12.8$ & $138.3 \pm 6.1$ & $137.80 \pm 8.5$ & $138.00 \pm 7.5$ \\
Potassium (meq/L) & $4.75 \pm 0.63$ & $4.61 \pm 0.53$ & $4.68 \pm 0.59$ & $4.34 \pm 0.47$ & $4.13 \pm 0.44$ & $4.22 \pm 0.47$ \\
Chloride (meq/L) & $98.2 \pm 8.2$ & $97.1 \pm 7.6$ & $97.7 \pm 7.9$ & $101.6 \pm 5.0$ & $102.4 \pm 4.6$ & $102.0 \pm 4.8$ \\
Calcium (mg/dL) & $9.15 \pm 1.11$ & $9.28 \pm 0.97$ & $9.21 \pm 1.05$ & $9.61 \pm 0.82$ & $9.42 \pm 1.02$ & $9.51 \pm 0.92$ \\
BUN (mg/dL) & $26.58 \pm 8.15$ & $24.29 \pm 7.43$ & $25.44 \pm 7.90$ & $21.03 \pm 11.62$ & $14.50 \pm 7.29$ & $17.50 \pm 10.05$ \\
Creatinine (mg/dL) & $0.84 \pm 0.167$ & $0.711 \pm 0.153$ & $0.781 \pm 0.174$ & $1.059 \pm 0.349$ & $0.757 \pm 0.219$ & $0.90 \pm 0.349$ \\
Uric acid (mg/dL) & $5.85 \pm 1.48$ & $4.61 \pm 1.21$ & $5.26 \pm 1.49$ & $5.65 \pm 1.23$ & $4.66 \pm 1.48$ & $5.12 \pm 1.45$
\end{tabular}

Within the hypertensive and normotensive groups, the mean levels of creatinine, BUN, uric acid and potassium were significantly higher in male compared to female $(\mathrm{P}<0.05)$. However, there were no significant differences in the levels of sodium, calcium and chloride $(p>0.05)$ between male and female within these two groups. While the mean levels of chloride, sodium, calcium and creatinine were significantly higher in the hypertensive group compared to normotensive $(\mathrm{p}<0.05)$, there was no significant difference between these two groups in the levels of uric acid ( $p>0.05$ ). Hypertensive individuals however had significantly lower mean levels of potassium and BUN $(p<0.05)$.

\section{DISCUSSION}

Abnormal lipid and lipoprotein levels are all positively associated with an increased risk in the incidence of $\operatorname{CVD}(21-23)$. In western Europe and the USA, CVD is the leading cause of morbidity and mortality with hypertension being the most common cause with a prevalence above $20 \%$ in the general population $(24,25)$. In many developing countries, serum lipid levels are low and the incidence of CVD is less frequent when compared to western countries
(26). However, these populations are increasingly exposed to urbanisation and associated changes in diet, physical activity and life styles. As a consequence, a CVD epidemic may be emerging due to an increase in risk factors. In the past four decades, the kingdom of Saudi Arabia has undergone profound socio-economic changes due to rapid urbanisation and a large increase in per capita income. This background suggests an increased prevalence of CVD which prompted this study (27). Information on CVD risk factors and hypertension in the population of the Eastern Province of Saudi Arabia is limited. Such information would allow us to improve control of hypertension which may contribute to a decrease in common causes of morbidity and mortality. The procedure by which a cohort is selected for any study is of fundamental importance for the interpretation of the results obtained and their accuracy. In the present study, which was part of a population based cross-sectional household survey, utmost care was taken to ensure the randomness of the sample selected and that all criteria were strictly adhered to. This broad coverage was needed for variables of genetic identity, physical environment and social and economical factors which were expected to affect the results. Thus the design of the study was 
similar to those perfomed in the U.S and European countries and therefore allows for meaningful comparison.

Although a number of studies have shown significant differences in the levels of haemoglobin, $\mathrm{RBC}, \mathrm{MCV}, \mathrm{HCT}$ and $\mathrm{MCH}$ between hypertensive and normotensive individuals, other studies have shown no significant differences between these groups (28-32). Our data do not show any significant differences in the levels of these parameters between hypertensive and the normotensive control group. However, the mean levels of haemoglobin, WBC and platelets were significantly higher in the hypertensive group compared to the normotensive. Furthermore, it has to be emphasised that there is a significant difference in the parameters between the gender within each group. This is in agreement with previous population based studies (33).

The present study shows that serum concentrations of Tc, LDL-c, HDL-C, Tg, glucose and HbAI-c were significantly higher in the hypertensive group compared to controls. This was true not only among men but also among women and is in close agreement with previous reported data (6). High glucose and HbAIc levels are a reflection of higher prevalence of diabetes mellitus among hypertensives as compared to normotensives (34). There is a relatively small but significant increase in the level of HDL-c, which is an important measure to compare across populations in hypertensive individuals compared to normotensive counterparts and in female compared to male. This conclusion is at variance with an earlier study of HDL-c in Saudi male and female (7). However, the observed higher levels of HDL-c in women modifies the coronary heart disease risk factors in this population. On comparison of lipid profile results obtained with the results of the European or American cohort, it is clear that Saudi populations in the Eastern Province have on average a lower lipid concentration. This can be reflected by the lower prevalence of CVD (10\%) (unpublised data) seen in the region compared to European or American populations $(>20 \%)(35)$.

It has been well established that an increased level of sodium and a decreased level of potassium is associated with hypertension $(36,37)$. Our data clearly indicate that the hypertensive group has a higher level of sodium and a lower level of potassium compared with the control normotensive group. This is true in other populations with a high prevalence of hypertension which may be due to hypertensive medication (38). In addition the data clearly indicate that hypertensive individuals have a higher mean creatinine level compared to normotensive individuals. This agrees with previous published data for other populations. This suggests some degree of renal impairment in this population. In this regard, it is unusual that the blood urea nitrogen was lower in hypertensives than in normotensives.

In conclusion, the present data have made an important contribution in establishing base line values of serum lipids in normal and hypertensive populations in the Eastern Province. In addition, these data address the feasibility of reducing the serum lipids of hypertensives in order to reduce the prevalence of CVD.

\section{ACKNOWLEDGEMENTS}

To King Abdul Aziz City for Science and Technology for funding this project. Our thanks are also extended to the field investigators and secretarial assistants.

\section{REFERENCES}

1. Tunstall-Pedoe, H., Kuulasmaa, K., Mahonen, M., et al. Contribution of trends in survival and coronary events rates to changes in coronary heart disease mortality.: 10 years results from 37 WHO Monica Project populations. Monitoring trends and determinants in cardiovascular disease. Lancet. 1999; 353: 1547.

2. Worthley, S. G., Osende, J. L., Helft, G. et al. Coronary artery disease: Pathogenesis and acute coronary syndromes. Mt. Sinai J. Med. 2001; 68: 167.

3. Hertzer, T., Schlinzig, T., Krobo, K., et al. Endothelial dysfunction, oxidative stress and risk of cardiovascular events in patients with coronary artery disease. Circulation. 2001; 104: 2673.

4. Zieske, A. W., Malcolm, G. T. and Strong, S. Natural history and risk of atherosclerosis in children and youth: the PDAY study. Pediatric Pathol. Mol. Med. 2002; 21: 213.

5. Parhami, A. D., Morrow, J. and Balucan, et al. Lipid oxidation products have opposite effects on calcifying vascular cell and bone cell differentiation: a possible explanation for the paradox of calcification in asteoporotic patients. Arterioseler thromb Vas. Biol. 1997; 17: 680.

6. Al-Nuaim, A. R. High prevalence of metabolic risk factors for CVD among Saudi population, aged 30-64 years. Int. J. Cardiol. 1997a; 19: 227.

7. Al-Nuaim, A. R. Serum total and fractionated cholesterol distribution and prevalence of hypercholesterolemia in urban and rural communites in Saudi Arabia. Int. J. Cardiol. 1997b; 58: 141.

8. Cooper, R., Cutler, J., Desvignes Nickens, P., et al. Trends and disparities in coronary heart disease, stroke and other cardiovascular diseases in the United States: finding of the National Conference on Cardiovascular Disease Prevention. Circulation 2000; 102: 3137.

9. Ahmed, A. F. and Mahmoud, M. E. The prevalence of hypertension in Saudi Arabia. Saudi Med. J. 1992; 13: 548.

10. Shanks, N. J., Kennedy, M. and Al-Kalal. D. Prevalence of hypertension in Saudi Arabia. The Practitioner, East Mediterranean Edition. 1996; 735.

11. Al-Shammari, S. A., Ali, M., Al-Shammari, A., et al. Blood lipid concentration and other cardiovascular risk factors among Saudis. Fam. Pract. 1994; 11: 153.

12. Al-Nozha, M. M., Ali, M. S. and Osman, A. K. Arterial hypertension in Saudi Arabia. Ann. Saudi Med. 1997; 17: 170.

13. Abalkhail, B. A., Shawky, S., Ghabrab, Z. T. M. et al. Hypercholesterolemia and 5-year risk of development of coronary heart disease among university and school workers in Jeddah, Saudi Arabia. Prev. Med. 2000; 31: 390.

14. Uddin, K. N. Prevalence of hypertension in Saudi Arabia. The practitioner, East Mediterranean Edition. 1994; 805. 
15. Al-Rajeh, S., Awada, A., Miazi, G. and Larbi, E. Stroke in a Saudi Arabian national guard community: Analysis of 500 consecutive cases from a population based hospital. Stroke. 1993; 24: 1635.

16. The sixth report of the Joint National Committee on prevention, detection, evaluation and treatment of high blood pressure. Arch. Intern. Med. 1997; 157: 2413.

17. Trinder, P. Determination of blood glucose using oxidase peroxidase system with a non-carcinogenic chromogene. $J$. Clin. Pathol. 1968; 22: 158.

18. Allain, C. C., Poon L. S., Chan, C. S. et al. Enzymatic determiantion of total serum cholesterol. Clin. Chem. 1974; 20: 470 .

19. Burtis, C. and Ashwood, E. R. Fundamentals of Clinical Chemistry. 5th Edition W. B. Saunders Company. Pp. 486.

20. Friedewald, W. L., Levy, R. I. and Fredrickson, D. S. Estimation of the concentration of low density lipoprotein cholesterol in plasma without use of the preparative ultracentrifuge. Clin. Chem. 1972; 18: 499.

21. Neaton, J. D., Blackburn, H., Jacobs, D., et al. Serum cholesterol level and mortality findings for men screened in multiple risk factor intervention trial. Arch. Int. Med. 1992; 152: 1490.

22. The scandinavian semivistatin survival study group. Randomized trial of cholesterol lowering in 4,444 patients with coronary heart disease. The Scandinavian survival study (4S). Lancet. 1994; 344: 1383.

23. The long term intervention with pravastatin in aschemic disease (lipid) study group. Prevention of cardiovascular events and death with pravastatin in patients with coronary heart disease and a broad based of initial cholesterol levels. N. Engl. J. Med. 1998; 339: 1349.

24. Rowland, M. and Roberts, J. NCHS Advance Data. Vital and health statistics of the National Centre for Health Statistics. Np 84, October 8, 1982. Washington D.C., US Department of Health and Human Services.

25. Burt, V. L., Cytler, J. A., Higgins, M., et al. Trends in the prevalence, awareness, treatment and control of hypertension in the adult US population. Data from the Health Examination Survey 1960-1991. Hypertension. 1995; 26: 305 .
26. Zhou, B., Rao. X., Dennis, B. H, et al. The relationship between dietary factors and serum lipid in Chinese urban and rural populations of Beijing and Guangzhou PRC-USA cardiovascular and cardiopulmonary research group. Int. J. Epidemiol. 1995; 24: 528.

27. Osman, A. K. and Al-Nozha, M. M. Risk factors of coronary artery diseases in different regions of Saudi Arabia. Eastern Mediterranian Health J. 2000; 6: 354.

28. Strazzullo, P., Cappuccio, F. P., Lacoviello, L., et al. Erythrocyte volume and blood pressure in a cross sectional population based study. J. Hypertens. 1990; 8: 179.

29. Cirillo, M., Laurenzi, M., Trevisan, M. and Stanley, J. Hematocrit blood pressure and hypertension. The Gubbio population study. Hypertension. 1992; 20: 314.

30. Bruschi, G., Minari, M., Bruschi, M. E., et al. Similarities of essential and spontaneous hypertension: volume and number of blood cells. Hypertension. 1986; 8: 983.

31. Sharp, D. S., Curb, J. D., Schatz, I. J., et al. Mean red cell volume as a correlate of blood pressure. Circulation. 1996; 93: 1677

32. Spencer, C. G. C., Gurney, D., Blann, A. D., et al. Von Willebrand factor, soluble p-selection and target organ damage in hypertension: a substudy of the AngloScandinavian cardiac outcomer trial. Hypertension. 2002; 40: 61 .

33. Woodward, M., Rumely, A., Tunstall-Pedoe, H. et al. Associations of blood theology and interleukin-6 with cardiovascular risk factors and prevalent cardiovascular diseases. Brit. J. Haematol. 1999; 104: 246.

34. Skarfors, E. T., Selinus, K. L. and Lithel, H. Risk factors for developing non-insulin dependent diabetes: a 10 year follow up of men in Uppsala. Brit. Med. J. 1991; 303: 755.

35. Jiang, H. and Whelton, P. K. Epidemiology and prevention of hypertension. Medical Clinics of North America. 1997; 1077.

36. Geleijnse, J. M., Kok, F. J. and Grobbee, D. E. Blood pressure response to changes in sodium and potassium intake: a metaregression analysis of randomized trials. J. Hum. Hypertens. 2003; 17: 471.

37. Hooper, L., Bartlett, C., Davey Smith, G. and Ebrahim, S. Reduced dietary salt for prevention of cardiovascular disease. Cochrane Data Base Syst. Rev. 2003; (3): CD003656. 